\title{
STRIPPING AND CONJUGATION IN THE MOD $p$ STEENROD ALGEBRA AND ITS DUAL
}

\author{
DAGMAR M. MEYER
}

(communicated by Hvedri Inassaridze)

\begin{abstract}
Let $p$ be an odd prime and $\mathcal{A}^{*}$ the mod $p$ Steenrod algebra. We study the technique known as "stripping" applied to $\mathcal{A}^{*}$ and derive certain conjugation formulas both for $\mathcal{A}^{*}$ and its dual, generalising work of J. H. Silverman for $p=2$ ("Conjugation and excess in the Steenrod algebra", Proc. Am. Math. Soc. 119 (1993), no.2, 657 - 661; "Hit polynomials and conjugation in the dual Steenrod algebra", Math. Proc. Camb. Philos. Soc. 123 (1998), no.3, 531 - 547) to the case of an odd prime.
\end{abstract}

\section{Introduction and statement of results}

In this note we study the technique known as "stripping" applied to the mod $p$ Steenrod algebra $\mathcal{A}^{*}$, where $p$ is an odd prime, and use the results obtained to prove certain conjugation formulas both in $\mathcal{A}^{*}$ and its dual. This generalises work of Judith Silverman carried out in [S1] and $[\mathbf{S 3}]$ for $p=2$ to the case of an odd prime. More precisely, our results concern Steenrod operations which lie in the sub-Hopf algebra $\mathcal{P}^{*}$ of $\mathcal{A}^{*}$ which is generated by the reduced power operations $\mathrm{P}(i), i \geqslant 1$, in dimensions $|\mathrm{P}(i)|=2 i(p-1)$. We use the convention $\mathrm{P}(0):=1$.

Of particular interest are the Steenrod operations in $\mathcal{P}^{*}$ which are of the form

$$
\mathrm{P}[k ; f]:=\mathrm{P}\left(p^{k-1} f\right) \cdot \mathrm{P}\left(p^{k-2} f\right) \cdot \ldots \cdot \mathrm{P}(p f) \cdot \mathrm{P}(f)
$$

where $k \geqslant 1$ and $f \geqslant 0$. Note that $\mathrm{P}[1 ; f]$ is just $\mathrm{P}(f)$. Being a sub-Hopf algebra, $\mathcal{P}^{*}$ inherits the canonical anti-automorphism $\chi$ of $\mathcal{A}^{*}$; following notation introduced in $[\mathbf{W} \mathbf{W}]$, we write $\hat{\theta}$ instead of $\chi(\theta)$. In particular, $\hat{\mathrm{P}}(a)=\chi(\mathrm{P}(a))$ and $\hat{\mathrm{P}}[k ; f]=\chi(\mathrm{P}[k ; f])$.

For $m \geqslant 0$ we define

$$
\gamma(m):=\sum_{i=0}^{m-1} p^{i} .
$$

Our first main result is an explicit conjugation formula for $\mathrm{P}[k ; f]$ in certain special cases. It generalises Thm. 3.1 in $[\mathbf{S 1}]$ to odd primes:

Theorem 4.6 For all positive integers $s, t$ and $c$ with $1 \leqslant c \leqslant p$ the following conjugation formula holds:

$$
\hat{\mathrm{P}}[s ; c \gamma(t)]=(-1)^{s t c} \mathrm{P}[t ; c \gamma(s)]
$$

The main result concerning conjugation in the dual $\mathcal{P}_{*}$ is a conjugation formula for certain elements $\mathcal{X}_{I}(k)$, which are defined in Section 5. This formula is the mod $p$ analogue of

During the preparation of this article the author was supported by a "bourse post-doctorale Alfred Kastler" of the French Ministry of Foreign Affairs.

Received 11 October 1999; published on 24 January 2000.

1991 Mathematics Subject Classification: 55S10

Key words and phrases: stripping, conjugation, Steenrod algebra, antiautomorphism

(C) 2000, Dagmar M. Meyer. Permission to copy for private use granted. 
Prop. 5.5 in [S3]. A special case states that modulo monomials of length strictly greater than $k$ the operations $\hat{\xi}_{i}^{\gamma(k)}$ and $(-1)^{i k} \xi_{k}^{\gamma(i)}$ coincide up to a certain error term; the conjugate of the error term is a sum of monomials of length strictly greater than $i$ :

Theorem 5.6 Let $i, k>0$. Modulo monomials of length $>k$ we have

$$
\hat{\xi}_{i}^{\gamma(k)} \equiv(-1)^{i k} \xi_{k}^{\gamma(i)}-\sum_{\operatorname{Id}_{k} \neq \tau \in \mathfrak{S}(k)} \operatorname{sign}(\tau) \prod_{j=0}^{k-1} \hat{\xi}_{i+\tau(j)-j}^{p^{j}} .
$$

Here $\mathfrak{S}(k)$ denotes the symmetric group acting on $\{0,1,2, \ldots, k-1\}$ and $\hat{\xi}_{r}:=0$ for $r<0$.

In particular, if $f<\gamma(k+1)$ is a non-negative integer then

$$
\hat{\xi}_{k}^{\gamma(i)} \cap \mathrm{P}[i ; f]=(-1)^{i k} \xi_{i}^{\gamma(k)} \cap \mathrm{P}[i ; f]=(-1)^{i k} \mathrm{P}[i ; f-\gamma(k)],
$$

where we use the notation $y \cap_{-}$for the stripping operation $D(y)$.

The ideas underlying the proofs of the results in this paper are similar to those of their mod 2 counterparts in $[\mathbf{S 1}]$ and $[\mathbf{S 3}]$. However, getting down to the details we note two major differences that appear in the odd-primary case: first of all, in just about every formula we prove there are some signs involved, and secondly (in Section 4) we have to deal with mod $p$ binomial coefficients which appear as non-trivial coefficients in our formulas. These difficulties cause the generalisation of the mod 2 results to be not quite as straightforward as it may seem at first glance.

Both Thm. 4.6 and Thm. 5.6 are essential ingredients for the work carried out in $[\mathbf{M}]$. There the Steenrod operations $\hat{\mathrm{P}}[k ; f]$ are studied further; in particular the excess of these operations is determined. In fact, that project was one of the main motivations for the work on the problems discussed in the present paper.

Acknowledgements: I would like to thank Judith Silverman for many helpful comments on the subject of this paper, in particular for supplying the elegant proof of Lemma 4.4 which is reproduced here. I also thank Ken Monks for pointing out to me the work of Ismet Karaca on related problems and for sending me copies of $[\mathbf{K a 2}],[\mathbf{K a 3}]$.

\section{Preliminaries}

Let $\mathcal{S}$ denote the additive monoid of sequences of non-negative integers almost all of which are 0 , with componentwise addition. We write $0_{\mathcal{S}}$ for the trivial element. Throughout we shall use capital letters to denote sequences in $\mathcal{S}$ and small letters for their coordinates; e.g. $S=$ $\left(s_{1}, s_{2}, \ldots\right)$. If $S$ has $s_{i}=0$ for $i>L$, we write $S$ as $\left(s_{1}, s_{2}, \ldots, s_{L}\right)$. The degree of an element $S \in \mathcal{S}$ is defined to be $|S|=\sum_{i \geqslant 1} s_{i}\left(p^{i}-1\right)$, its length as $\operatorname{len}(S)=\min \left\{i \geqslant 0 \mid s_{j}=0 \forall j>i\right\}$, and its excess as $\operatorname{ex}(S)=\sum_{i \geqslant 1} s_{i}$. It will be convenient to adjoin an extra element $*$ to $\mathcal{S}$ with the property that $*+x=x+*=*$ for all $x \in \mathcal{S} \cup\{*\}=: \mathcal{S}^{*}$. We also define sequences $B(j)$ for any $j \in \mathbb{Z}$ : if $j \geqslant 0$ then $B(j)$ is the sequence with $b(j)_{i}:=\delta_{i j}$, if $j<0$ we set $B(j):=*$.

There are many interesting bases for $\mathcal{A}^{*}$ and hence for $\mathcal{P}^{*}$; the most important and most commonly used are the basis of admissible monomials ("admissible basis") and the Milnor basis. Recall that the monomial $\mathrm{P}\left(a_{1}\right) \cdot \ldots \cdot \mathrm{P}\left(a_{n}\right)$ with $a_{n}>0$ is admissible if $a_{r} \geqslant p a_{r+1}$ for all $1 \leqslant r<n$; we also define $P(0)=1$ to be admissible. The admissible basis of $\mathcal{P}^{*}$ can be parameterised in terms of the numbers $s_{i}=a_{i}-p a_{i+1}$; that is, given a sequence $S \in \mathcal{S}$ of length $n>0$, we define the admissible element $E[S]:=\mathrm{P}\left(a_{1}\right) \cdot \ldots \cdot \mathrm{P}\left(a_{n}\right)$ by setting $a_{n}=s_{n}$ and $a_{i}=p a_{i+1}+s_{i}$ for $1 \leqslant i \leqslant n-1$. We also set $E\left[0_{\mathcal{S}}\right]:=P(0)=1$. For example, if $S=(0, \ldots, 0, f)$ has length $k$ then $E[S]=\mathrm{P}\left(p^{k-1} f\right) \cdot \ldots \cdot \mathrm{P}(f)=\mathrm{P}[k ; f]$.

For the Milnor basis of $\mathcal{P}^{*}$ consider the dual Hopf algebra $\mathcal{P}_{*}$. This is a polynomial algebra over $\mathbb{F}_{p}$ on generators $\xi_{i}(i \geqslant 1)$ in dimension $2\left(p^{i}-1\right)$; we use the convention $\xi_{0}:=1$. For 
$S \in \mathcal{S}$ we write $\xi[S]$ for the monomial $\prod_{i \geqslant 1} \xi_{i}^{s_{i}}$. In particular, $\xi\left[B_{j}\right]=\xi_{j}$ for any $j \geqslant 0$. The Milnor basis of $\mathcal{P}^{*}$ itself is the basis dual to the basis of $\mathcal{P}_{*}$ consisting of all the monomials $\xi[S]$ with $S \in \mathcal{S}$; the element dual to $\xi[S]$ will be denoted by $M[S]$.

We further set $M[*]=0=E[*]$ and $\xi[*]=0$, and we adopt the convention that $M[S]=$ $0=E[S]$ and $\xi[S]=0$ if $S$ is a finite sequence of integers which does not belong to $\mathcal{S}$, i.e. with at least one negative entry. In particular, $\xi_{i}:=0$ if $i<0$.

For any $S \in \mathcal{S}$ we define length and excess of the monomial $\xi[S]$ as $\operatorname{len}(S)$ and $2 \operatorname{ex}(S)$ respectively. Likewise, for the admissible and the Milnor basis we define

$$
\begin{aligned}
\operatorname{len}_{E}(E[S]) & :=\operatorname{len}(S)=: \operatorname{len}_{M}(M[S]), \\
\operatorname{ex}_{E}(E[S]) & :=2 \operatorname{ex}(S)=: \operatorname{ex}_{M}(M[S]) .
\end{aligned}
$$

More generally, suppose $\theta$ is any homogeneous element of $\mathcal{P}^{*}$ with a basis representation given by $\theta=\sum_{i=1}^{n} \alpha_{i} B\left[S_{i}\right]$, where $B$ stands for either $E$ or $M$. Then we set

$$
\begin{aligned}
\operatorname{len}_{B}(\theta) & :=\max _{i}\left\{\operatorname{len}_{B}\left(B\left[S_{i}\right]\right)\right\}=\max _{i}\left\{\operatorname{len}\left(S_{i}\right)\right\} \\
\operatorname{ex}_{B}(\theta) & :=\min _{i}\left\{\operatorname{ex}_{B}\left(B\left[S_{i}\right]\right)\right\}=2 \min _{i}\left\{\operatorname{ex}\left(S_{i}\right)\right\} .
\end{aligned}
$$

The excess of any operation $\theta$ in $\mathcal{P}^{*}$ can also be defined as $\operatorname{ex}(\theta):=\min \left\{n \mid \theta\left(\iota_{n}\right) \neq\right.$ $\left.0 \in H^{*}\left(K(\mathbb{Z} / p, n) ; \mathbb{F}_{p}\right)\right\}$, where $\iota_{n} \in H^{*}\left(K(\mathbb{Z} / p, n) ; \mathbb{F}_{p}\right)$ is the fundamental class. In fact, all the different definitions of excess that we have given coincide (cf. $[\mathbf{K r}])$; in particular $\operatorname{ex}_{E}(\theta)=\operatorname{ex}(\theta)=\operatorname{ex}_{M}(\theta)$.

By [Mi], the change-of-basis matrix in each dimension between the admissible and the Milnor basis is upper triangular with diagonal entry \pm 1 , if for both bases we use the order induced by the right-lexicographical order on $\mathcal{S}$. From this it follows that for any $S \in \mathcal{S}$ we have $\operatorname{len}_{E}(M[S])=\operatorname{len}_{E}(E[S])=\operatorname{len}(S)$ and $\operatorname{len}_{M}(E[S])=\operatorname{len}_{M}(M[S])=\operatorname{len}(S)$, and one easily sees that this implies $\operatorname{len}_{M}(\theta)=\operatorname{len}_{E}(\theta)$ for any $\theta \in \mathcal{P}^{*}$. Henceforth we denote this common value simply by len $(\theta)$.

\section{3. $\quad$ Stripping in $\mathcal{P}^{*}$}

\subsection{Recollections about the stripping technique}

Much recent progress on problems related to the structure of the Steenrod algebra has been made by applying a tool that has become known as "stripping technique" (for a detailed account see $[\mathbf{W}])$. This technique applies to any Hopf algebra, so in particular to the cocommutative, connected Hopf algebra $\mathcal{P}^{*}$.

Let $\Delta^{*}$ denote the diagonal map of $\mathcal{P}^{*}$ and $\langle$,$\rangle the inner product. We consider the natural$ action of the dual Hopf algebra $\mathcal{P}_{*}$ on $\mathcal{P}^{*}$ which is given for each $\xi \in \mathcal{P}_{*}$ by

$$
D(\xi): \mathcal{P}^{*} \stackrel{\Delta^{*}}{\longrightarrow} \mathcal{P}^{*} \otimes \mathcal{P}^{*} \stackrel{\text { id } \otimes\langle\xi,\rangle}{\longrightarrow} \mathcal{P}^{*} ;
$$

this action satisfies

$$
\langle\xi \cdot \psi, \theta\rangle=\langle\psi, D(\xi) \theta\rangle
$$

for all $\psi \in \mathcal{P}_{*}, \theta \in \mathcal{P}^{*}$. The operation $D(\xi): \mathcal{P}^{*} \longrightarrow \mathcal{P}^{*}$ is called "stripping by $\xi$ " and can be considered as a kind of cap-product. For this reason the notation

$$
D(\xi) \theta=: \xi \cap \theta
$$

has become customary.

For the reader's convenience we now recall some important properties of the stripping operation (cf. [S2]):

Let $\Delta_{*}$ denote the product of $\mathcal{P}_{*}$ and $\phi_{*}$ the comultiplication; the canonical anti-automorphism of $\mathcal{P}_{*}$ will again be denoted by $\chi$, with $\chi(y)=: \hat{y}$. In what follows let $\phi_{*}(y)=: \sum y^{\prime} \otimes y^{\prime \prime}$ and

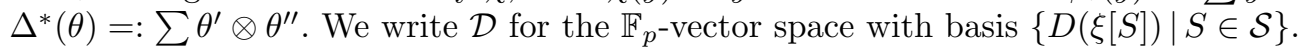


The maps $\chi: \mathcal{P}_{*} \longrightarrow \mathcal{P}_{*}, \Delta_{*}: \mathcal{P}_{*} \otimes \mathcal{P}_{*} \longrightarrow \mathcal{P}_{*}$ and $\phi_{*}: \mathcal{P}_{*} \longrightarrow \mathcal{P}_{*} \otimes \mathcal{P}_{*}$ induce maps

$$
\begin{aligned}
\chi: \mathcal{D} \longrightarrow \mathcal{D}, & D(y) \mapsto D(\hat{y}) \\
\Delta_{*}: \mathcal{D} \otimes \mathcal{D} \longrightarrow \mathcal{D}, & D\left(y_{1}\right) \otimes D\left(y_{2}\right) \mapsto D\left(y_{1} \cdot y_{2}\right) \\
\phi_{*}: \mathcal{D} \longrightarrow \mathcal{D} \otimes \mathcal{D}, & D(y) \mapsto \sum D\left(y^{\prime}\right) \otimes D\left(y^{\prime \prime}\right) .
\end{aligned}
$$

Proposition 3.1. The following formulas hold:
1. $\left(y_{1}+y_{2}\right) \cap \theta=y_{1} \cap \theta+y_{2} \cap \theta$
2. $\left(y_{1} \cdot y_{2}\right) \cap \theta=\left(y_{2} \cdot y_{1}\right) \cap \theta=y_{1} \cap\left(y_{2} \cap \theta\right)=y_{2} \cap\left(y_{1} \cap \theta\right)$
3. $y \cap\left(\theta_{1} \cdot \theta_{2}\right)=\sum\left(y^{\prime} \cap \theta_{1}\right) \cdot\left(y^{\prime \prime} \cap \theta_{2}\right)$
4. $\hat{y} \cap\left(\theta_{1} \cdot \theta_{2}\right)=\sum\left(\widehat{y^{\prime \prime}} \cap \theta_{1}\right) \cdot\left(\widehat{y^{\prime}} \cap \theta_{2}\right)$
5. $\hat{y} \cap \hat{\theta}=\widehat{y \cap \theta}$

\subsection{Stripping in the Milnor basis and in the admissible basis}

The effect of stripping by an element $y \in \mathcal{P}_{*}$ on a Milnor basis element can easily be described by writing $y$ as a sum of basis elements $\xi[R]$. In fact, recall that the comultiplication $\Delta^{*}$ of $\mathcal{P}^{*}$ is determined by the formula

$$
\Delta^{*}(M[S])=\sum_{S^{\prime}+S^{\prime \prime}=S} M[S] \otimes M\left[S^{\prime \prime}\right]
$$

([Mi]). From this and the definition of stripping one easily sees that

$$
\xi[R] \cap M[S]=M[S-R] .
$$

In particular, stripping does not increase length.

Determining the effect of $D(\xi[R])$ on a given admissible monomial is more involved. More generally, let $\mathrm{P}\left(a_{1}\right) \cdots \mathrm{P}\left(a_{n}\right)$ be any (not necessarily admissible) monomial in $\mathcal{P}^{*}$. For $n \geqslant k$, we define $\mathcal{V}_{n, k}$ to be the set of all sequences $\left(v_{1}, \ldots, v_{n}\right)$ in which the non-zero elements form exactly the subsequence $\left(p^{k-1}, \ldots, p, 1\right)$. For example, $\mathcal{V}_{3,2}$ consists of $(0, p, 1),(p, 0,1)$, and $(p, 1,0)$. For $n<k$, we define $\mathcal{V}_{n, k}:=\emptyset$.

Proposition 3.2. With this notation

$$
\xi_{k} \cap\left(\mathrm{P}\left(a_{1}\right) \cdot \ldots \cdot \mathrm{P}\left(a_{n}\right)\right)=\sum_{V \in \mathcal{V}_{n, k}} \mathrm{P}\left(a_{1}-v_{1}\right) \cdot \ldots \cdot \mathrm{P}\left(a_{n}-v_{n}\right) .
$$

Proof. The proof is analogous to that of Prop. 3.1 in [S3]. Alternatively, see [CWW, Section 2].

We note the following consequences:

Corollary 3.3. 1. If $\theta \in \mathcal{P}^{*}$ has length $n$, then $\xi[S] \cap \theta=0$ for any $S \in \mathcal{S}$ of length greater than $n$; in particular $\xi_{k} \cap \theta=0$ for any $k>n$.

2. If $\mathrm{P}\left(a_{1}\right) \cdot \ldots \cdot \mathrm{P}\left(a_{k}\right)$ is admissible of excess $2 e$, then

$$
\xi_{k} \cap\left(\mathrm{P}\left(a_{1}\right) \cdot \ldots \cdot \mathrm{P}\left(a_{k}\right)\right)=\mathrm{P}\left(a_{1}-p^{k-1}\right) \cdot \mathrm{P}\left(a_{2}-p^{k-2}\right) \cdot \ldots \cdot \mathrm{P}\left(a_{k}-1\right),
$$

which is again admissible and has excess $2 e-2$. Consequently, if $R=\left(r_{1}, \ldots, r_{k}\right) \in \mathcal{S}$, then $\xi_{k} \cap E[R]=E\left[\left(r_{1}, \ldots, r_{k-1}, r_{k}-1\right)\right]$.

3. In particular,

$$
\xi_{k} \cap \mathrm{P}[k ; f]=\mathrm{P}[k ; f-1] \quad \text { and } \quad \hat{\xi}_{k} \cap \hat{\mathrm{P}}[k ; f]=\hat{\mathrm{P}}[k ; f-1],
$$


where the second equation follows from Prop. 3.1(5).

The next thing we determine is the action of $D(\widehat{\xi[R]})$ on a given element $\theta \in \mathcal{P}^{*}$. By $[\mathbf{M i}]$, conjugation in $\mathcal{P}_{*}$ is determined by

$$
\hat{\xi}_{k}=\sum_{\alpha \in \operatorname{Part}(k)}(-1)^{l(\alpha)} \prod_{i=1}^{l(\alpha)} \xi_{\alpha_{i}}^{p^{\sigma_{i}(\alpha)}}
$$

where $\alpha$ runs through all ordered partitions $\alpha=\left(\alpha_{1}, \alpha_{2}, \ldots, \alpha_{l(\alpha)}\right)$ of $k, l(\alpha)$ is the length of the partition $\alpha$, and $\sigma_{i}(\alpha)$ is the partial sum $\sum_{j=1}^{i-1} \alpha_{j}$.

Consequences 3.4. 1. The excess of $\xi_{k}=\xi\left[B_{k}\right]$ is 2 for any $k$, so the summand with the largest excess in formula (2) is the monomial corresponding to the partition $\alpha$ of length $l(\alpha)=k$ with $\alpha_{i}=1$ for $1 \leqslant i \leqslant k$, i.e. the summand

$$
(-1)^{k} \prod_{i=1}^{k} \xi_{1}^{p^{i-1}}=(-1)^{k} \xi_{1}^{\gamma(k)}
$$

which has excess $2 \gamma(k)$. Hence stripping by $\hat{\xi}_{k}$ reduces excess by no more than $2 \gamma(k)$.

2. Since $\xi_{l} \cap \mathrm{P}(f)=0$ for all $l>1$, we have

$$
\begin{aligned}
\hat{\xi}_{k} \cap \mathrm{P}(f) & =(-1)^{k} \xi_{1}^{\gamma(k)} \cap \mathrm{P}(f) \\
& = \begin{cases}(-1)^{k} \mathrm{P}(f-\gamma(k)) & \text { if } f \geqslant \gamma(k) \\
0 & \text { otherwise. }\end{cases}
\end{aligned}
$$

3.3. Stripping $\mathrm{P}[\Lambda ; f]$ by $\hat{\xi}_{k}^{j}$

We will be mostly concerned with the special Steenrod operations $\mathrm{P}[\Lambda ; f]$. Therefore we take a closer look at the action of the stripping operations $D\left(\hat{\xi}_{k}^{j}\right)$ on these elements.

Lemma 3.5. For any $\theta$ in $\mathcal{P}^{*}$ we have

$$
\hat{\xi}_{k} \cap(\mathrm{P}[2 ; f] \cdot \theta)=\mathrm{P}(p f) \cdot\left(\hat{\xi}_{k} \cap(\mathrm{P}(f) \cdot \theta)\right) .
$$

Proof. The proof is analogous to the proof of Lemma 4.4 in [S2]: recall that the comultiplication in $\mathcal{P}_{*}$ is given by

$$
\phi_{*}\left(\xi_{k}\right)=\sum_{j=0}^{k} \xi_{k-j}^{p^{j}} \otimes \xi_{j}
$$

([Mi]). Hence by Prop. 3.1(3) we obtain

$$
\hat{\xi}_{k} \cap(\mathrm{P}[2 ; f] \cdot \theta)=\mathrm{P}(p f) \cdot\left(\hat{\xi}_{k} \cap(\mathrm{P}(f) \cdot \theta)\right)+\sum_{j=1}^{k}\left(\hat{\xi}_{j} \cap \mathrm{P}(p f)\right) \cdot\left(\hat{\xi}_{k-j}^{p^{j}} \cap(\mathrm{P}(f) \cdot \theta)\right) .
$$

Cons. 3.4(2) implies that $\hat{\xi}_{j} \cap \mathrm{P}(p f)=-\hat{\xi}_{j-1}^{p} \cap \mathrm{P}(p f-1)$, thus

$$
\begin{aligned}
\sum_{j=1}^{k}\left(\hat{\xi}_{j} \cap \mathrm{P}(p f)\right) & \cdot\left(\hat{\xi}_{k-j}^{j} \cap(\mathrm{P}(f) \cdot \theta)\right) \\
& =-\sum_{j=1}^{k}\left(\hat{\xi}_{j-1}^{p} \cap \mathrm{P}(p f-1)\right) \cdot\left(\left(\hat{\xi}_{(k-1)-(j-1)}^{p}\right)^{p^{j-1}} \cap(\mathrm{P}(f) \cdot \theta)\right) \\
& =-\hat{\xi}_{k-1}^{p} \cap(\mathrm{P}(p f-1) \cdot \mathrm{P}(f) \cdot \theta) .
\end{aligned}
$$


But by the Adem relations $\mathrm{P}(p f-1) \cdot \mathrm{P}(f)=0$, which proves the claim.

The following more general result is now easily proved by induction on $\Lambda$, the case $\Lambda=2$ being given by Lemma 3.5:

Proposition 3.6. For $\Lambda \geqslant 2$ and any $\theta$ in $\mathcal{P}^{*}$ we have

$$
\hat{\xi}_{k} \cap(\mathrm{P}[\Lambda ; f] \cdot \theta)=\mathrm{P}[\Lambda-1 ; p f] \cdot\left(\hat{\xi}_{k} \cap(\mathrm{P}(f) \cdot \theta)\right) .
$$

Finally, we investigate what happens if we strip $\mathrm{P}[\Lambda ; f]$ by $\hat{\xi}_{k}$ a total of $j$ times. We note that only the right-most $j$ places are affected:

Proposition 3.7. Suppose $\Lambda>j \geqslant 1$. Then

$$
\hat{\xi}_{k}^{j} \cap \mathrm{P}[\Lambda ; f]=\mathrm{P}\left[\Lambda-j ; p^{j} f\right] \cdot\left(\hat{\xi}_{k}^{j} \cap \mathrm{P}[j ; f]\right) .
$$

Proof. The proof is by induction on $j$, starting with $j=1$ where the result is provided by Prop. 3.6.

\section{Conjugation formulas for $\mathcal{P}^{*}$}

In this section we establish some useful formulas involving conjugation of elements in $\mathcal{P}^{*}$. In particular, we determine the simple formula for $\hat{\mathrm{P}}[s ; c \gamma(t)]$ with $1 \leqslant c \leqslant p$ that was announced in the introduction.

Suppose that $y$ is a non-negative integer. We use the notation $\alpha_{i}(y)$ for the coefficient of $p^{i}$ in the $p$-adic expansion of $y$, i.e. $y=: \sum_{i \geqslant 0} \alpha_{i}(y) p^{i}$.

The following lemma will be needed for the proof of Prop. 4.3.

Lemma 4.1. Suppose that $k, l, c, m$ and e are non-negative integers with

1. $k>l$,

2. $1 \leqslant c \leqslant p-1$,

3. $m<p^{k-1}$,

4. $m \equiv 0 \bmod p^{l}$.

Then the following relation mod $p$ holds:

$$
\left(\begin{array}{c}
c\left(p^{k}-p^{l}\right)+e \\
p m
\end{array}\right) \equiv-\sum_{i=1}^{c}\left(\begin{array}{c}
c \\
i
\end{array}\right)\left(\begin{array}{c}
c\left(p^{k}-p^{l}\right)+e \\
p m+i p^{l}
\end{array}\right)+\left(\begin{array}{c}
e \\
p m+c p^{l}
\end{array}\right)
$$

Proof. The proof relies on the fact that mod $p$ we have the relation $\left(\begin{array}{l}x \\ y\end{array}\right) \equiv \prod_{i \geqslant 0}\left(\begin{array}{l}\alpha_{i}(x) \\ \alpha_{i}(y)\end{array}\right)$. There are three cases: (I) $\alpha_{l}(e)=c$, (II) $0 \leqslant \alpha_{l}(e) \leqslant c-1$, and (III) $c+1 \leqslant \alpha_{l}(e) \leqslant p-1$. If we are in case (I) then the first term on the right of (4) is 0 and

$$
\left(\begin{array}{c}
c\left(p^{k}-p^{l}\right)+e \\
p m
\end{array}\right) \equiv\left(\begin{array}{c}
e \\
p m+c p^{l}
\end{array}\right)
$$

as required.

If we are in case (II) then the second term on the right of (4) is zero and so we have to show that

$$
\left(\begin{array}{c}
c\left(p^{k}-p^{l}\right)+e \\
p m
\end{array}\right) \equiv-\sum_{i=1}^{c}\left(\begin{array}{l}
c \\
i
\end{array}\right)\left(\begin{array}{c}
c\left(p^{k}-p^{l}\right)+e \\
p m+i p^{l}
\end{array}\right)
$$


i.e. that

$$
1 \equiv-\sum_{i=1}^{c}\left(\begin{array}{l}
c \\
i
\end{array}\right)\left(\begin{array}{c}
p-c+\alpha_{l}(e) \\
i
\end{array}\right)
$$

for $0 \leqslant \alpha_{l}(e) \leqslant c-1$. Setting $a:=p-c+\alpha_{l}(e)$ this amounts to showing that

$$
\sum_{i=0}^{c}\left(\begin{array}{l}
c \\
i
\end{array}\right)\left(\begin{array}{l}
a \\
i
\end{array}\right) \equiv 0
$$

for all $p-c \leqslant a \leqslant p-1$. In order to show this equivalence, note that

$$
\sum_{i=0}^{c}\left(\begin{array}{l}
c \\
i
\end{array}\right)\left(\begin{array}{l}
a \\
i
\end{array}\right) \equiv \sum_{i=0}^{c}\left(\begin{array}{l}
c \\
i
\end{array}\right)\left(\begin{array}{c}
a \\
a-i
\end{array}\right) \equiv\left(\begin{array}{c}
c+a \\
c
\end{array}\right)
$$

as one sees by considering the coefficient of $x^{c}$ in the binomial expansion of $(x+1)^{c+a}=$ $(x+1)^{c}(x+1)^{a}$. Now the claim follows since $\left(\begin{array}{c}c+a \\ c\end{array}\right) \equiv 0$ for $p-c \leqslant a \leqslant p-1$.

Case (III) is similar.

We will need the following multiplication formulas:

Lemma 4.2. Let $u$ and $v$ be non-negative integers. Then

$$
\mathrm{P}(u) \cdot \hat{\mathrm{P}}(v)=(-1)^{v} \sum_{R}\left(\begin{array}{c}
|R|+\operatorname{ex}(R) \\
p u
\end{array}\right)_{p} M[R]
$$

and

$$
\hat{\mathrm{P}}(u) \cdot \mathrm{P}(v)=(-1)^{u} \sum_{R}\left(\begin{array}{c}
\mathrm{ex}(R) \\
v
\end{array}\right)_{p} M[R]
$$

where the sum ranges over all sequences $R$ in $\mathcal{S}$ with $|R|=(p-1)(u+v)$ and ()$_{p}$ denotes mod p binomial coefficients.

Proof. The proof of (6) can be found in $[\mathbf{G}]$. The other equality, (7), can be extracted from [Ka1].

Remark. In [Ka1], our Lemma 4.2 is stated (wrongly) without any minus signs. Unfortunately, Karaca does not explicitly say what his definition of $\hat{\mathrm{P}}(u)$ is. Instead, for the special Milnor basis elements $M\left[\left(0, \ldots, 0, r_{t}=p^{s}\right)\right]=: \mathrm{P}_{t}^{s}$ he defines $\widehat{\mathrm{P}_{t}^{s}}$ as $(-1)^{s} \chi\left(\mathrm{P}_{t}^{s}\right)$. Since there exists a basis of $\mathcal{P}^{*}$ which consists of certain monomials in elements of the form $\mathrm{P}_{t}^{s}$, it is possible to figure out what the expression $\hat{\mathrm{P}}(u)$ should mean according to Karaca's definition, assuming that $\widehat{\mathrm{P}_{t}^{s} \cdot \mathrm{P}_{u}^{v}}:=\widehat{\mathrm{P}_{u}^{v}} \cdot \widehat{\mathrm{P}_{t}^{s}}$. However, doing this translation one easily sees that there should be some non-trivial coefficients in his formula. The correct result can nevertheless easily be deduced from the argument given in [Ka1].

After these preparations we are in a position to prove the following "hat-passing formula", which is a slightly generalised odd prime version of the formula given in [S1, Lemma 2.3]:

Proposition 4.3. Suppose that $k, l, c, m$ and $n$ are non-negative integers with
1. $k>l$,
2. $1 \leqslant c \leqslant p-1$,
3. $m+n=c p^{l} \gamma(k-l)$,
4. $m<p^{k-1}$,
5. $m \equiv 0 \bmod p^{l}$. 
We use the convention $\hat{\mathrm{P}}(s):=0$ if $s<0$. Then for $l=0$ we have

$$
\mathrm{P}(m) \cdot \hat{\mathrm{P}}(n)=(-1)^{c} \hat{\mathrm{P}}(m+n-p m-c) \cdot \mathrm{P}(p m+c)
$$

and for $l>0$ we have

$$
\begin{aligned}
\mathrm{P}(m) \cdot \hat{\mathrm{P}}(n) & =\sum_{i=1}^{c}(-1)^{i+1}\left(\begin{array}{l}
c \\
i
\end{array}\right)_{p} \mathrm{P}\left(m+i p^{l-1}\right) \cdot \hat{\mathrm{P}}\left(n-i p^{l-1}\right) \\
& +(-1)^{c} \hat{\mathrm{P}}\left(m+n-p m-c p^{l}\right) \cdot \mathrm{P}\left(p m+c p^{l}\right) .
\end{aligned}
$$

Proof. In order to see that for $l=0$ only one term in the expression for $\mathrm{P}(m) \cdot \hat{\mathrm{P}}(n)$ appears, note that $|R|=(p-1) c \gamma(k)=c\left(p^{k}-1\right)$, so that by applying Equation (6) in Lemma 4.2 we obtain

$$
\mathrm{P}(m) \cdot \hat{\mathrm{P}}(n)=(-1)^{n} \sum_{|R|=c\left(p^{k}-1\right)}\left(\begin{array}{c}
c\left(p^{k}-1\right)+\operatorname{ex}(R) \\
p m
\end{array}\right)_{p} M[R] .
$$

Now recall that $\operatorname{ex}(R)=\sum_{i \geqslant 1} r_{i}$. Dividing $|R|$ by $(p-1)$ and substituting ex $(R)-\sum_{i \geqslant 2} r_{i}$ for $r_{1}$ we have

$$
c \gamma(k)=\frac{|R|}{p-1}=\sum_{i \geqslant 1} r_{i} \gamma(i)=\operatorname{ex}(R)+\sum_{i \geqslant 2} r_{i} p \gamma(i-1) .
$$

Thus we see that $\operatorname{ex}(R) \equiv c \bmod p$. Now we apply Lemma 4.1 with $e=\operatorname{ex}(R)$; we have just seen that we are always in case (I) so that

$$
\left(\begin{array}{c}
c\left(p^{k}-1\right)+\operatorname{ex}(R) \\
p m
\end{array}\right) \equiv\left(\begin{array}{c}
\operatorname{ex}(R) \\
p m+c
\end{array}\right) .
$$

Equation (7) in Lemma 4.2 now implies that

$$
\begin{aligned}
\mathrm{P}(m) \cdot \hat{\mathrm{P}}(n) & =(-1)^{n} \sum_{|R|=c\left(p^{k}-1\right)}\left(\begin{array}{c}
c\left(p^{k}-1\right)+\operatorname{ex}(R) \\
p m
\end{array}\right)_{p} M[R] \\
& =(-1)^{c}(-1)^{m+n-p m-c} \sum_{|R|=c\left(p^{k}-1\right)}\left(\begin{array}{c}
\operatorname{ex}(R) \\
p m+c
\end{array}\right)_{p} M[R] \\
& =(-1)^{c} \hat{\mathrm{P}}(m+n-p m-c) \cdot \mathrm{P}(p m+c) .
\end{aligned}
$$

The formula for $l>0$ easily follows from Lemma 4.1, carefully keeping track of any minus signs that enter into the formula.

In order to arrive at the simple description of $\hat{\mathrm{P}}[s ; c \gamma(t)]$ that will be obtained in Theorem 4.6 we need yet another lemma. The elegant proof given here, due to Judith Silverman, is a nice application of the "stripping technique" discussed in Section 3 and replaces the original, more complicated proof which didn't use stripping at all.

Lemma 4.4. Let $c$ and $l$ be positive integers with $1 \leqslant c \leqslant p-1$. Then $\mathrm{P}(c \gamma(l)) \cdot \mathrm{P}\left(a p^{l-1}\right)=0$ for any a which satisfies $p-c \leqslant \alpha_{0}(a) \leqslant p-1$.

Proof. The lemma is proved by downward induction on $c$. We start with the case $c=p-1$ so that $1 \leqslant \alpha_{0}(a) \leqslant p-1$. Then by the Adem relations we have

$$
\begin{aligned}
\mathrm{P}\left(p^{l}-1\right) & \cdot \mathrm{P}\left(a p^{l-1}\right) \\
& =\sum_{z=0}^{p^{l-1}}(-1)^{p^{l}-1+z}\left(\begin{array}{c}
(p-1)\left(a p^{l-1}-z\right)-1 \\
p^{l}-1-p z
\end{array}\right)_{p} \mathrm{P}\left(p^{l}-1+a p^{l-1}-z\right) \cdot \mathrm{P}(z) .
\end{aligned}
$$


We show that the mod $p$ binomial coefficients appearing in this formula are all 0 . First consider the case $z=0$ : since $1 \leqslant \alpha_{0}(a) \leqslant p-1$ we have $0 \leqslant \alpha_{l-1}\left((p-1) a p^{l-1}-1\right) \leqslant p-2$, but $\alpha_{l-1}\left(p^{l}-1\right)=p-1$ and so $\left(\begin{array}{c}(p-1) a p^{l-1}-1 \\ p^{l}-1\end{array}\right) \equiv 0$. On the other hand, if $z \neq 0$ then there exists some index $j_{0}$ with $0 \leqslant j_{0} \leqslant l-2$ such that $1 \leqslant z_{j_{0}} \leqslant p-1$ but $z_{j}=0$ for all $0 \leqslant j<j_{0}$. Hence $1 \leqslant \alpha_{j_{0}}((p-1) z)=p-z_{j_{0}} \leqslant p-1$ and so $0 \leqslant \alpha_{j_{0}}\left((p-1)\left(a p^{l-1}-z\right)-1\right) \leqslant p-2$. But $\alpha_{j_{0}}\left(p^{l}-1-p z\right)=p-1$ and so again $\left(\begin{array}{c}(p-1)\left(a p^{l-1}-z\right)-1 \\ p^{l}-1-p z\end{array}\right) \equiv 0$.

Now let $1 \leqslant c<p-1$ and suppose that the lemma has been shown to be true for all $\hat{c}$ with $c<\hat{c} \leqslant p-1$. Choose $a$ with $p-c \leqslant \alpha_{0}(a) \leqslant p-1$ (which implies $p-(c+1) \leqslant \alpha_{0}(a-1) \leqslant p-1$ and $\left.p-(c+1) \leqslant \alpha_{0}(a) \leqslant p-1\right)$. The lemma for $c+1$ guarantees that

$$
\mathrm{P}((c+1) \gamma(l)) \cdot \mathrm{P}\left(a p^{l-1}\right)=0
$$

and

$$
\mathrm{P}((c+1) \gamma(l)) \cdot \mathrm{P}\left((a-1) p^{l-1}\right)=0 .
$$

Using Equation (3), Prop. 3.1(4) and Cons. 3.4(2) we strip Equation (8) by $\hat{\xi}_{l}$ to obtain

$$
\begin{aligned}
0 & =\hat{\xi}_{l} \cap\left[\mathrm{P}((c+1) \gamma(l)) \cdot \mathrm{P}\left(a p^{l-1}\right)\right] \\
= & {\left[\hat{\xi}_{l} \cap \mathrm{P}((c+1) \gamma(l))\right] \cdot \mathrm{P}\left(a p^{l-1}\right) } \\
& \quad+\sum_{i=0}^{l-1}\left[\hat{\xi}_{i} \cap \mathrm{P}((c+1) \gamma(l))\right] \cdot\left[\hat{\xi}_{l-i}^{p^{i}} \cap \mathrm{P}\left(a p^{l-1}\right)\right] \\
& =(-1)^{l} \mathrm{P}(c \gamma(l)) \cdot \mathrm{P}\left(a p^{l-1}\right)+E,
\end{aligned}
$$

where $E$ is defined to be the big sum in (10). It remains to show that $E=0$. We fix $i$ with $1 \leqslant i \leqslant l-1$ and observe that for any $b \geqslant 0$ we have

$$
\hat{\xi}_{l-i}^{p^{i}} \cap \mathrm{P}(b)=(-1)^{l-i} \mathrm{P}\left(b-p^{i} \gamma(l-i)\right)=-\hat{\xi}_{l-i-1}^{p^{i}} \cap \mathrm{P}\left(b-p^{l-1}\right) .
$$

Setting $b=a p^{l-1}$, we find that $E$ can be rewritten as

$$
\begin{aligned}
E & =-\sum_{i=0}^{l-1}\left[\hat{\xi}_{i} \cap \mathrm{P}((c+1) \gamma(l))\right] \cdot\left[\hat{\xi}_{l-i-1}^{p^{i}} \cap \mathrm{P}\left((a-1) p^{l-1}\right)\right] \\
& =-\hat{\xi}_{l-1} \cap\left[\mathrm{P}((c+1) \gamma(l)) \cdot \mathrm{P}\left((a-1) p^{l-1}\right)\right] .
\end{aligned}
$$

But by (9), the product in (11) is 0 . Consequently $E=0$ as desired.

The next lemma establishes the basis of induction for Theorem 4.6.

Lemma 4.5. Let $c$ be an integer with $1 \leqslant c \leqslant p-1$. Then

$$
\hat{\mathrm{P}}(c \gamma(s))=(-1)^{s c} \mathrm{P}[s ; c] .
$$

Proof. The case $s=1$ is clear: by [Mi] we have

$$
\hat{\mathrm{P}}(c)=(-1)^{c} \sum_{|Q|=c(p-1)} M[Q]=(-1)^{c} \mathrm{P}(c),
$$

and in general

$$
\hat{\mathrm{P}}(c \gamma(s))=(-1)^{c \gamma(s)} \sum_{|Q|=c\left(p^{s}-1\right)} M[Q] .
$$


By induction and Equation (6) we obtain

$$
\begin{aligned}
(-1)^{s c} \mathrm{P}[s ; c] & =(-1)^{s c} \mathrm{P}\left(p^{s-1} c\right) \cdot \mathrm{P}[s-1 ; c] \\
& =(-1)^{c} \mathrm{P}\left(p^{s-1} c\right) \cdot \hat{\mathrm{P}}(c \gamma(s-1)) \\
& =(-1)^{c \gamma(s)} \sum_{|R|=c\left(p^{s}-1\right)}\left(\begin{array}{c}
|R|+\operatorname{ex}(R) \\
c p^{s}
\end{array}\right)_{p} M[R],
\end{aligned}
$$

so that by (12) it only remains to show that $\left(\begin{array}{c}|R|+\operatorname{ex}(R) \\ c p^{s}\end{array}\right) \equiv 1$ for all $R$ with $|R|=c\left(p^{s}-1\right)$. It follows directly from the definitions that $0 \leqslant \operatorname{ex}(R) \leqslant \frac{|R|}{p-1}=c \gamma(s)$. On the other hand it is easy to see that the sequence $\left(0, \cdots, 0, r_{s}=c\right)$ is of excess $c$ and that this is the minimal excess of any sequence in $\mathcal{S}$ of degree $c\left(p^{s}-1\right)$. The inequality $c \leqslant \operatorname{ex}(R) \leqslant c \gamma(s)$ now implies that

$$
c p^{s} \leqslant|R|+\operatorname{ex}(R) \leqslant c p \gamma(s)=c p^{s}+c p^{s-1}+\ldots+c p
$$

so that indeed $\left(\begin{array}{c}|R|+\operatorname{ex}(R) \\ c p^{s}\end{array}\right) \equiv 1$ for all $R$ with $|R|=c\left(p^{s}-1\right)$.

Finally we can prove the conjugation formula announced earlier on, which is a slightly generalised mod $p$ version of [S1, Theorem 3.1]. The proof is similar to the one in the mod 2 case.

Theorem 4.6. For all positive integers $s, t$ and $c$ with $1 \leqslant c \leqslant p$ the following conjugation formula holds:

$$
\hat{\mathrm{P}}[s ; c \gamma(t)]=(-1)^{s t c} \mathrm{P}[t ; c \gamma(s)]
$$

Proof. We first prove the theorem for $1 \leqslant c \leqslant p-1$. The case $c=p$ will follow from the case $c=1$ by a stripping argument.

The proof for $1 \leqslant c \leqslant p-1$ is by induction on $t$. The basis of induction (i.e. the case $t=1$ or equivalently $s=1$ ) has been established in Lemma 4.5. So let us assume that $t>1, s>1$ and that the theorem has been shown to be true for all $1 \leqslant \hat{t} \leqslant t-1$, all $s$ and also for $\hat{t}=t$, all $1 \leqslant \hat{s} \leqslant s-1$. We begin with the following remark:

Remark. Under the above assumptions the following is true:

For all non-negative integers a with $p-c \leqslant \alpha_{0}(a) \leqslant p-1$ and for all $1 \leqslant l<s$ we have

$$
\hat{\mathrm{P}}\left(a p^{l-1}\right) \cdot \mathrm{P}[l ; c \gamma(t)]=0 .
$$

We prove this result as follows: we have

$$
\hat{\mathrm{P}}\left(a p^{l-1}\right) \cdot \mathrm{P}[l ; c \gamma(t)]=\chi\left[\hat{\mathrm{P}}[l ; c \gamma(t)] \cdot \mathrm{P}\left(a p^{l-1}\right)\right],
$$

which by induction equals

$$
\left.(-1)^{t l c} \chi\left[\mathrm{P}[t ; c \gamma(l)] \cdot \mathrm{P}\left(a p^{l-1}\right)\right]=(-1)^{t l c} \chi[\mathrm{P}[t-1 ; p c \gamma(l))] \cdot \mathrm{P}(c \gamma(l)) \cdot \mathrm{P}\left(a p^{l-1}\right)\right] .
$$

But by Lemma 4.4 the expression $\mathrm{P}(c \gamma(l)) \cdot \mathrm{P}\left(a p^{l-1}\right)$ vanishes. This proves the remark.

Now we get back to the proof of the theorem: by induction we obtain

$$
\begin{aligned}
\hat{\mathrm{P}}[t ; c \gamma(s)] & =\chi(\mathrm{P}[t-1 ; c \gamma(s)]) \cdot \chi\left(\mathrm{P}\left(p^{t-1} c \gamma(s)\right)\right) \\
& =(-1)^{(t-1) s c} \mathrm{P}[s ; c \gamma(t-1)] \cdot \hat{\mathrm{P}}\left(p^{t-1} c \gamma(s)\right) .
\end{aligned}
$$

We claim that for $1 \leqslant d \leqslant s$ the following formula holds:

$$
\mathrm{P}[d ; c \gamma(t-1)] \cdot \hat{\mathrm{P}}\left(p^{t-1} c \gamma(s)\right)=(-1)^{d c} \hat{\mathrm{P}}\left(p^{t+d-1} c \gamma(s-d)\right) \cdot \mathrm{P}[d ; c \gamma(t)]
$$

Proof of the claim: for $d=1$ we have to show that

$$
\mathrm{P}(c \gamma(t-1)) \cdot \hat{\mathrm{P}}\left(p^{t-1} c \gamma(s)\right)=(-1)^{c} \hat{\mathrm{P}}\left(p^{t} c \gamma(s-1)\right) \cdot \mathrm{P}(c \gamma(t)) .
$$


This follows immediately from Prop. 4.3 with $m=c \gamma(t-1), n=p^{t-1} c \gamma(s), k=t+s-1$ and $l=0$. So suppose that $2 \leqslant d \leqslant s$, assuming that the claim has been proved for all $1 \leqslant \hat{d}<d$. Then using induction we obtain

$$
\begin{aligned}
\mathrm{P}[d ; c \gamma & (t-1)] \cdot \hat{\mathrm{P}}\left(p^{t-1} c \gamma(s)\right) \\
\quad & =\mathrm{P}\left(p^{d-1} c \gamma(t-1)\right) \cdot \mathrm{P}[d-1 ; c \gamma(t-1)] \cdot \hat{\mathrm{P}}\left(p^{t-1} c \gamma(s)\right) \\
& =(-1)^{(d-1) c} \mathrm{P}\left(p^{d-1} c \gamma(t-1)\right) \cdot \hat{\mathrm{P}}\left(p^{t+d-2} c \gamma(s-d+1)\right) \cdot P[d-1 ; c \gamma(t)] .
\end{aligned}
$$

Again, we apply Prop. 4.3, this time to the first two terms, with the parameters $m=p^{d-1} c \gamma(t-$ $1), n=p^{t+d-2} c \gamma(s-d+1), k=t+s-1$ and $l=d-1$. We deduce that

$$
\begin{aligned}
\mathrm{P}\left(p^{d-1} c \gamma(t-1)\right) \cdot \hat{\mathrm{P}}\left(p^{t+d-2} c \gamma(s-d+1)\right) & \\
= & \sum_{i=1}^{c}(-1)^{i+1}\left(\begin{array}{c}
c \\
i
\end{array}\right)_{p} \mathrm{P}\left(p^{d-1} c \gamma(t-1)+i p^{d-2}\right) \cdot \hat{\mathrm{P}}\left(\left(p^{t+d-2} c \gamma(s-d+1)-i p^{d-2}\right)\right. \\
& +(-1)^{c} \hat{\mathrm{P}}\left(p^{t+d-1} c \gamma(s-d)\right) \cdot \mathrm{P}\left(p^{d-1} c \gamma(t)\right) .
\end{aligned}
$$

By the remark, the terms in the big sum vanish upon multiplication with $\mathrm{P}[d-1 ; c \gamma(t)]$ from the right, and so we arrive at

$$
\begin{aligned}
\mathrm{P}[d ; c \gamma(t-1)] & \cdot \hat{\mathrm{P}}\left(p^{t-1} c \gamma(s)\right) \\
& =(-1)^{d c} \hat{\mathrm{P}}\left(p^{t+d-1} c \gamma(s-d)\right) \cdot \mathrm{P}\left(p^{d-1} c \gamma(t)\right) \cdot \mathrm{P}[d-1 ; c \gamma(t)] \\
& =(-1)^{d c} \hat{\mathrm{P}}\left(p^{t+d-1} c \gamma(s-d)\right) \cdot \mathrm{P}[d ; c \gamma(t)]
\end{aligned}
$$

which proves the claim.

Setting $d=s$ and substituting back into expression (13) yields

$$
\begin{aligned}
\hat{\mathrm{P}}[t ; c \gamma(s)] & =(-1)^{(t-1) s c} \mathrm{P}[s ; c \gamma(t-1)] \cdot \hat{\mathrm{P}}\left(p^{t-1} c \gamma(s)\right) \\
& =(-1)^{t s c} \mathrm{P}[s ; c \gamma(t)]
\end{aligned}
$$

which finishes the proof of the theorem for $1 \leqslant c \leqslant p-1$.

There remains the case $c=p$. We strip the formula

$$
\hat{\mathrm{P}}[s ; \gamma(t+1)]=(-1)^{s(t+1)} \mathrm{P}[t+1 ; \gamma(s)]
$$

(this is the case $c=1$ with $t+1$ instead of $t$ ) by $\hat{\xi}_{s}$, and by Cor. 3.3(3) we obtain

$$
\begin{aligned}
\hat{\mathrm{P}}[s ; p \gamma(t)] & =\hat{\xi}_{s} \cap \hat{\mathrm{P}}[s ; \gamma(t+1)] \\
& =(-1)^{s(t+1)} \hat{\xi}_{s} \cap \mathrm{P}[t+1 ; \gamma(s)]
\end{aligned}
$$

which by Cons. 3.4(2) and Prop. 3.6 equals

$$
\begin{aligned}
(-1)^{s(t+1)} \mathrm{P}[t ; p \gamma(s)] \cdot\left(\hat{\xi}_{s} \cap \mathrm{P}(\gamma(s))\right) & =(-1)^{s(t+1)} \mathrm{P}[t ; p \gamma(s)] \cdot(-1)^{s} \mathrm{P}(0) \\
& =(-1)^{s t} \mathrm{P}[t ; p \gamma(s)] .
\end{aligned}
$$

This completes the proof of the theorem.

We observe the following:

Corollary 4.7. Let $s, t$ and $c$ be non-negative integers with $s \geqslant 1$ and $1 \leqslant c \leqslant p$. Then the operations $\hat{\mathrm{P}}[s ; c \gamma(t)]$ have length exactly $t$ independently of $s$ and $c$. More generally, if $\gamma(t) \leqslant f<\gamma(t+1)$ then the operations $\hat{\mathrm{P}}[s ; f]$ are all of length exactly $t$, independently of $s$.

Proof. For $t \geqslant 1$ the first statement is an immediate consequence of Theorem 4.6; for $t=0$ the statement is trivial. The second statement follows since stripping operations cannot increase length (cf. Section 3.2). 


\section{Conjugation formulas for $\mathcal{P}_{*}$}

We now turn to conjugation in the dual Steenrod algebra. Let $\mathfrak{S}(k)$ be the symmetric group with identity $\operatorname{Id}_{k}$ acting on $\{0,1,2, \ldots, k-1\}$. For $\tau \in \mathfrak{S}(k)$ and $i \geqslant 0$ we define

$$
\begin{aligned}
Z_{i}(k ; \tau) & :=\sum_{j=0}^{k-1} p^{j} B(i+\tau(j)-j), \\
X_{i}(k ; \tau) & :=\xi\left[Z_{i}(k ; \tau)\right]=\prod_{j=0}^{k-1} \xi_{i+\tau(j)-j}^{p^{j}},
\end{aligned}
$$

and

$$
\mathcal{X}_{i}(k):=\sum_{\tau \in \mathfrak{S}(k)} \operatorname{sign}(\tau) X_{i}(k ; \tau)
$$

Observation 5.1. $Z_{i}\left(k ; \operatorname{Id}_{k}\right)=\gamma(k) B(i)$ and $X_{i}\left(k ; \operatorname{Id}_{k}\right)=\xi_{i}^{\gamma(k)}$.

We will need the following lemma:

Lemma 5.2. For $k \geqslant 1$ we have $\mathcal{X}_{1}(k)=(-1)^{k} \hat{\xi}_{k}$.

Proof. The proof is by induction on $k$. Let $k=1$, then $\mathcal{X}_{1}(1)=\xi_{1}=-\hat{\xi}_{1}$, so the assertion is true in this case. Now suppose the statement has been shown to be true for all $1 \leqslant \hat{k}<k$. Note that if $X_{1}(k ; \tau) \neq 0$ then necessarily $\tau(j) \geqslant j-1$ for all $j$. So if $X_{1}(k ; \tau) \neq 0$ then define $l$ by $l=\tau^{-1} k-1$. If $l=k-1$ then we obtain a cycle decomposition of $\tau$ as $(k-1) \sigma$ for some $\sigma \in \mathfrak{S}(k-1)$. If $l \neq k-1$ then we obtain $\tau(k-1)=k-2, \tau(k-2)=k-3, \ldots, \tau(l+1)=l$, so that $\tau$ has a cycle decomposition as $(k-1, k-2, \ldots, l) \sigma$ for some $\sigma \in \mathfrak{S}(l)$. In any case we have

$$
X_{1}(k ; \tau)=X_{1}(l ; \sigma) \cdot \xi_{k-l}^{p^{l}} .
$$

So for $0 \leqslant l \leqslant k-1$ let $\mathfrak{S}_{l}(k)=\{\tau \in \mathfrak{S}(k) \mid \tau(l)=k-1\}$; obviously $\mathfrak{S}(k)=\bigcup \mathfrak{S}_{l}(k)$. Then by induction

$$
\begin{aligned}
\mathcal{X}_{1}(k) & =\sum_{l=0}^{k-1} \sum_{\tau \in \mathfrak{S}_{l}(k)} \operatorname{sign}(\tau) X_{1}(k ; \tau) \\
& =\sum_{l=0}^{k-1} \xi_{k-l}^{p^{l}} \cdot \sum_{\sigma \in \mathfrak{S}(l)}(-1)^{k-1-l} \operatorname{sign}(\sigma) X_{1}(l ; \sigma) \\
& =(-1)^{k-1} \sum_{l=0}^{k-1} \xi_{k-l}^{p^{l}} \cdot \hat{\xi}_{l}=(-1)^{k} \hat{\xi}_{k},
\end{aligned}
$$

where in the last line we used Milnor's recursive formula for the anti-automorphism.

In analogy to $[\mathbf{S 3}]$ we make the following more general definitions:

Definition 5.3. For $k \geqslant 1$, let $\mathcal{I}(k)$ be the set of non-decreasing sequences $\left(i_{0}, i_{1}, \ldots, i_{k-1}\right)$ of positive integers. For $\tau \in \mathfrak{S}(k)$ and $I \in \mathcal{I}(k)$ we define

$$
\begin{aligned}
& Z_{I}(k ; \tau):=\sum_{j=0}^{k-1} p^{j} B\left(i_{\tau(j)}+\tau(j)-j\right), \\
& X_{I}(k ; \tau):=\xi\left[Z_{I}(k ; \tau)\right]=\prod_{j=0}^{k-1} \xi_{i_{\tau(j)} p^{j}+\tau(j)-j}
\end{aligned}
$$


and

$$
\mathcal{X}_{I}(k):=\sum_{\tau \in \mathfrak{S}(k)} \operatorname{sign}(\tau) X_{I}(k ; \tau)
$$

We further define

$$
\begin{aligned}
& P_{I}(k ; \tau):=\sum_{j=0}^{k-1} p^{j+i_{0}} B\left(i_{\tau(j)}+\tau(j)-\left(j+i_{0}\right)\right), \\
& R_{I}(k ; \tau):=\xi\left[P_{I}(k ; \tau)\right]=\prod_{j=0}^{k-1} \xi_{i_{\tau(j)}+\tau(j)-\left(j+i_{0}\right)}^{p^{j+i_{0}}}
\end{aligned}
$$

and

$$
\mathcal{R}_{I}(k):=\sum_{\tau \in \mathfrak{S}(k)} \operatorname{sign}(\tau) R_{I}(k ; \tau) .
$$

Observations 5.4. 1. If $I=(i, i, \ldots, i) \in \mathcal{I}(k)$ is a constant sequence then we obtain $Z_{I}(k ; \tau)=Z_{i}(k ; \tau)$ and consequently $X_{I}(k ; \tau)=X_{i}(k ; \tau)$. Moreover, for such a sequence $I$ and $\tau \neq \operatorname{Id}_{k}$ we have $P_{I}(k ; \tau)=*$ and consequently $\mathcal{R}_{I}(k)=R_{I}\left(k ; \operatorname{Id}_{k}\right)=1$.

2. If $I=\left(i_{0}, i_{1}, \ldots, i_{k-1}\right) \in \mathcal{I}(k)$ and $i_{0}>1$ let $I[-1]$ denote the sequence $\left(i_{0}-1, i_{1}-\right.$ $\left.1, \ldots, i_{k-1}-1\right) \in \mathcal{I}(k)$. Then $\mathcal{R}_{I}(k)=\left(\mathcal{R}_{I[-1]}(k)\right)^{p}$.

Theorem 5.5. Let $k \geqslant 1$. Then $\hat{\mathcal{X}}_{I}(k) \equiv(-1)^{i_{0} k} \xi_{k}^{\gamma\left(i_{0}\right)} \cdot \hat{\mathcal{R}}_{I}(k)$ modulo monomials of length $>k$.

Proof. First recall that we have the following expression for $\hat{\mathcal{X}}_{I}(k)$ :

$$
\begin{aligned}
\hat{\mathcal{X}}_{I}(k) & =\sum_{\rho \in \mathfrak{S}(k)} \operatorname{sign}(\rho) \prod_{j=0}^{k-1} \hat{\xi}_{i_{\rho(j)}+\rho(j)-j}^{p^{j}} \\
& =\sum_{\rho \in \mathfrak{S}(k)} \operatorname{sign}(\rho) \hat{\xi}_{i_{\rho(0)}+\rho(0)} \cdot \prod_{j=1}^{k-1} \hat{\xi}_{i_{\rho(j)}+\rho(j)-j}^{p^{j}} .
\end{aligned}
$$

Applying Milnor's recursive formula for the anti-automorphism we obtain

$$
-\hat{\xi}_{i_{\rho(0)}+\rho(0)} \equiv \sum_{n=1}^{k} \xi_{n} \cdot \hat{\xi}_{i_{\rho(0)} p^{n}}
$$

modulo monomials of length $>k$. So we have

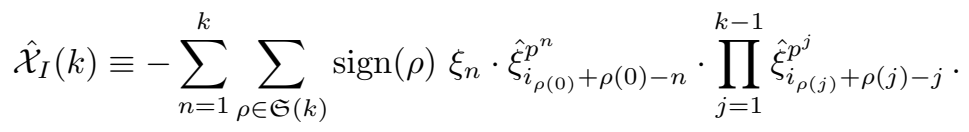

For each $\rho \in \mathfrak{S}(k)$ we define $\rho^{\prime}$ by

$$
\rho^{\prime}(l)= \begin{cases}\rho(0) & \text { if } l=k-1 \\ \rho(l+1) & \text { if } 0 \leqslant l \leqslant k-2 .\end{cases}
$$

Note that $\operatorname{sign}(\rho)=(-1)^{k-1} \operatorname{sign}\left(\rho^{\prime}\right)$. So

$$
\hat{\mathcal{X}}_{I}(k) \equiv(-1)^{k} \sum_{n=1}^{k} \sum_{\rho^{\prime} \in \mathfrak{S}(k)} \operatorname{sign}\left(\rho^{\prime}\right) \xi_{n} \cdot \hat{\xi}_{i_{\rho^{\prime}(k-1)}+\rho^{\prime}(k-1)-n}^{p^{n}} \cdot \prod_{l=0}^{k-2} \hat{\xi}_{i_{\rho^{\prime}(l)}+\rho^{\prime}(l)-(l+1)}^{p^{l+1}}
$$

modulo monomials of length $>k$. 
For the proof of the theorem, we fix $k$ and use induction on $i_{0}$. First suppose that $i_{0}=1$. Then

$$
\xi_{k} \cdot \hat{\mathcal{R}}_{I}(k)=\sum_{\tau \in \mathfrak{S}(k)} \operatorname{sign}(\tau) \xi_{k} \cdot \hat{\xi}_{i_{\tau(k-1)}}^{p^{k}+\tau(k-1)-k} \cdot \prod_{j=0}^{k-2} \hat{\xi}_{i_{\tau(j)}}^{p^{j+1}}+\tau(j)-(j+1)
$$

so that

$$
\begin{aligned}
& \hat{\mathcal{X}}_{I}(k)-(-1)^{k} \xi_{k} \cdot \hat{\mathcal{R}}_{I}(k) \\
& \equiv(-1)^{k} \sum_{n=1}^{k-1} \sum_{\rho^{\prime} \in \mathfrak{S}(k)} \operatorname{sign}\left(\rho^{\prime}\right) \xi_{n} \cdot \hat{\xi}_{i_{\rho^{\prime}(k-1)}}^{p^{n}}+\rho^{\prime}(k-1)-n \cdot \prod_{l=0}^{k-2} \hat{\xi}_{i_{\rho^{\prime}(l)}}^{p^{l+1}}+\rho^{\prime}(l)-(l+1)
\end{aligned}
$$

It can easily be verified that the summand in (14) associated to $n$ and $\rho^{\prime}$ is the negative of the term associated to $n$ and $\rho^{\prime \prime}$ where

$$
\rho^{\prime \prime}(l)= \begin{cases}\rho^{\prime}(l) & \text { if } l \neq n-1 \text { and } l \neq k-1 \\ \rho^{\prime}(n-1) & \text { if } l=k-1 \\ \rho^{\prime}(k-1) & \text { if } l=n-1\end{cases}
$$

(note that $\left.\operatorname{sign}\left(\rho^{\prime}\right)=-\operatorname{sign}\left(\rho^{\prime \prime}\right)\right)$. So the difference $\hat{\mathcal{X}}_{I}(k)-(-1)^{k} \xi_{k} \cdot \hat{\mathcal{R}}_{I}(k)$ vanishes modulo monomials of length $>k$ and the theorem holds for $i_{0}=1$.

The proof for general $I$ is similar. By induction we can assume that the statement is true for $\left(i_{0}-1, i_{1}-1, \ldots, i_{k}-1\right)=I[-1]$. By Observation $5.4(2)$

$$
\xi_{k}^{\gamma\left(i_{0}\right)} \cdot \hat{\mathcal{R}}_{I}(k)=\left(\xi_{k}^{\gamma\left(i_{0}-1\right)} \cdot \hat{\mathcal{R}}_{I[-1]}(k)\right)^{p} \cdot \xi_{k}
$$

which modulo terms of length $>k$ is

$$
\begin{aligned}
& \equiv\left((-1)^{k\left(i_{0}-1\right)} \hat{\mathcal{X}}_{I[-1]}(k)\right)^{p} \cdot \xi_{k} \\
& =(-1)^{k\left(i_{0}-1\right)} \xi_{k} \cdot \sum_{\tau \in \mathfrak{S}(k)} \operatorname{sign}(\tau) \prod_{j=0}^{k-1} \hat{\xi}_{i_{\tau(j)}-1+\tau(j)-j}^{p^{j+1}}
\end{aligned}
$$

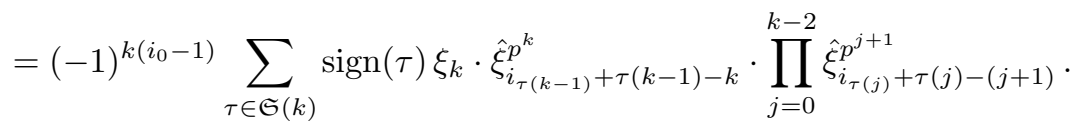

Now one can define $\rho^{\prime \prime}$ as before and proceed as in the case $i_{0}=1$ in order to establish the inductive step.

An especially interesting formula arises from Theorem 5.5 if we set $I=(i, i, \ldots, i)$, a constant sequence:

Theorem 5.6. Let $i, k>0$. Modulo monomials of length $>k$ we have

$$
\hat{\xi}_{i}^{\gamma(k)} \equiv(-1)^{i k} \xi_{k}^{\gamma(i)}-\sum_{\operatorname{Id}_{k} \neq \tau \in \mathfrak{S}(k)} \operatorname{sign}(\tau) \prod_{j=0}^{k-1} \hat{\xi}_{i+\tau(j)-j}^{p^{j}} .
$$

In particular, if $0 \leqslant f<\gamma(k+1)$ then

$$
\hat{\xi}_{k}^{\gamma(i)} \cap \mathrm{P}[i ; f]=(-1)^{i k} \xi_{i}^{\gamma(k)} \cap \mathrm{P}[i ; f]=(-1)^{i k} \mathrm{P}[i ; f-\gamma(k)] .
$$

Proof. The first part follows immediately from Theorem 5.5 and Observation 5.4(1), so it only remains to prove the second statement. By the part already proved we have the following equality:

$$
\hat{\xi}_{i}^{\gamma(k)} \cap \hat{\mathrm{P}}[i ; f]=(-1)^{i k} \xi_{k}^{\gamma(i)} \cap \hat{\mathrm{P}}[i ; f]-\left(\sum_{\operatorname{Id}_{k} \neq \tau \in \mathfrak{S}(k)} \operatorname{sign}(\tau) \prod_{j=0}^{k-1} \hat{\xi}_{i+\tau(j)-j}^{p^{j}}\right) \cap \hat{\mathrm{P}}[i ; f]
$$


Now observe that for any $\operatorname{Id}_{k} \neq \tau \in \mathfrak{S}(k)$ the product $\prod_{j=0}^{k-1} \xi_{i+\tau(j)-j}^{p^{j}}$ is of length strictly greater than $i$, so for any such $\tau$ we get

$$
\left(\prod_{j=0}^{k-1} \hat{\xi}_{i+\tau(j)-j}^{p^{j}}\right) \cap \hat{\mathrm{P}}[i ; f]=\chi\left[\left(\prod_{j=0}^{k-1} \xi_{s+\tau(j)-j}^{p^{j}}\right) \cap \mathrm{P}[i ; f]\right]=0 .
$$

Using Cor. 3.3(3) we thus obtain $\hat{\xi}_{i}^{\gamma(k)} \cap \hat{\mathrm{P}}[i ; f]=\hat{\mathrm{P}}[i ; f-\gamma(k)]=(-1)^{i k} \xi_{k}^{\gamma(i)} \cap \hat{\mathrm{P}}[i ; f]$. The claim now follows by application of $(-1)^{i k} \chi$ to this formula.

Finally, we note that Theorem 5.5 provides us with useful information regarding the behaviour of the stripping operations $D\left(\hat{\mathcal{X}}_{I}(k)\right)$ :

Corollary 5.7. 1. If $\operatorname{len}(\theta)<k$, then $\hat{\mathcal{X}}_{I}(k) \cap \theta=0$ for all $I \in \mathcal{I}(k)$.

2. If $\operatorname{len}(\theta)=k$, then $\hat{\mathcal{X}}_{I}(k) \cap \theta=(-1)^{i_{0} k} \hat{\mathcal{R}}_{I}(k) \cap\left(\xi_{k}^{\gamma\left(i_{0}\right)} \cap \theta\right)$.

3. In particular, $\hat{\mathcal{X}}_{I}(k) \cap \mathrm{P}[k ; f]=(-1)^{i_{0} k} \hat{\mathcal{R}}_{I}(k) \cap \mathrm{P}\left[k ; f-\gamma\left(i_{0}\right)\right]$.

Proof. This follows immediately from the theorem by invoking Prop. 3.1 and Cor. 3.3.

\section{References}

[CWW] D. P. Carlisle, G. Walker and R. M. W. Wood. The intersection of the admissible basis and the Milnor basis of the Steenrod algebra. J. Pure Appl. Algebra 128 (1998), no.1, 1-10

[G] A. M. Gallant. Excess and conjugation in the Steenrod algebra. Proc. Amer. Math. Soc. 76 (1979), no.1, 161-166

[Ka1] I. KaraCA. The nilpotence height of $P_{t}^{s}$ for odd primes. Trans. Amer. Math. Soc. 351 (1999), no.2, 547-558

[Ka2] I. KARACA. On the action of Steenrod operations on polynomial algebras. Turkish J. Math. 22 (1998), no.2, 163-170

[Ka3] I. KARACA. Conjugation in the $\bmod p$ Steenrod algebra and its dual. (preliminary version, oct. 1998)

[Kr] D. Kraines. On excess in the Milnor basis. Bull. London Math. Soc. 3 (1971), 363-365

[M] D. M. Meyer. Hit polynomials and excess in the $\bmod p$ Steenrod algebra. (prépublication numéro 1999-19, Université Paris-Nord)

[Mi] J. Milnor. The Steenrod algebra and its dual. Ann. of Math. (2) 67 (1958), $150-171$

[S1] J. H. Silverman. Conjugation and excess in the Steenrod algebra. Proc. Am. Math. Soc. 119 (1993), no.2, 657-661

[S2] J. H. Silverman. Stripping and conjugation in the Steenrod algebra. J. Pure Appl. Algebra 121 (1997), no.1, 95-106

[S3] J. H. Silverman. Hit polynomials and conjugation in the dual Steenrod algebra. Math. Proc. Camb. Philos. Soc. 123 (1998), no.3, 531-547

[WW] G. Walker And R. M. W. Wood. The nilpotence height of $\mathrm{Sq}^{2^{n}}$. Proc. Am. Math. Soc. 124 (1996), no.4, 1291-1295

[W] R. M. W. Wood. Problems in the Steenrod algebra. Bull. London Math. Soc. 30 (1998), no.5, 449-517 
This article may be accessed via WWW at http://www.rmi.acnet.ge/hha/ or by anonymous ftp at ftp://ftp.rmi.acnet.ge/pub/hha/volumes/2000/n1/n1.(dvi,ps,dvi.gz,ps.gz)

Dagmar M. Meyer dagmar@math.univ-paris13.fr meyerd@member.ams.org

LAGA, Institut Galilée, Univ. Paris-Nord 